\title{
Videogames for Emotion Regulation: A Systematic Review
}

\author{
Daniela Villani, PhD, Claudia Carissoli, PhD Candidate, Stefano Triberti, PhD, Antonella Marchetti, PhD,2 \\ Gabriella Gilli, PhD, and Giuseppe Riva, $\mathrm{PhD}^{1,3}$
}

\begin{abstract}
Introduction: Emotion regulation (ER) supports multiple individual functions and promotes mental health and wellbeing. Among the tools that may be used to help people in managing their affective states, videogames are reaching attention and are showing positive effects. Yet, little is known about their effectiveness.

Objective: This study aims to assess the amount and quality of studies investigating the effects and modalities of the use of videogames for ER.

Materials and Methods: A systematic literature search according to PRISMA guidelines was performed. Subsequently, according to expert advice other few studies have been added.

Results: Twenty-three studies met the inclusion criteria and were included in the review; they can be categorized into three groups, namely (1) cross-sectional and qualitative studies, (2) experimental studies investigating the effects of videogame experience on ER and (3) ER intervention with serious games.

Discussion: Discussion of the reviewed studies highlights that frequent gaming with commercial games offers more opportunities for ER improvement (related to gameplay and enjoyment of fictional properties) than limited-time experiences, such as those supported by bespoke serious games. This research area is still in its infancy and findings need to be interpreted with caution; furthermore, future reviews are encouraged to include clinical populations.

Conclusion: Videogames offer several opportunities for ER and a challenge for educational and psychological interventions.
\end{abstract}

Keywords: Emotion regulation, Videogames, Serious games, Systematic review, Mental health

\section{Introduction}

F ROM THE EARLY 1990s, there has been exponential growth in research on emotion regulation (ER) in the field of psychology. ER consists in "the extrinsic and intrinsic processes responsible for monitoring, evaluating, and modifying emotional reactions, especially their intensive and temporal features, to accomplish one's goals."1(p27-28) One of the most widely used model aimed to describe ER is the socalled "process model" of $\mathrm{ER}^{2,3}$ that distinguishes among different strategies mostly classified by the time at which they intervene in the emotion generation process (namely antecedent-focused and response-focused processes). In this article we consider a broad conception of ER that entails the regulation of all emotionally charged states, including mood and positive or negative effect. This is coherent with Koole's classification $^{4}$ of ER strategies that also includes their functions, namely the fact that people aim to achieve certain psychological outcomes by regulating their emotions. Specifically outcomes are related to the satisfaction of hedonic needs (possible ER strategies to reach this aim are thinking pleasurable or relaxing thoughts), facilitation of specific goals and tasks (e.g., effortful distraction and cognitive reappraisal), and maintenance of the integrity of their overall personality system (e.g., mindfulness training and relaxation exercises).

The literature shows that effective ER leads to several important outcomes, such as mental health, ${ }^{5}$ subjective and psychological wellbeing, ${ }^{6}$ and relationship satisfaction. ${ }^{7}$ On the contrary, a great deal of research highlighted the role of emotion dysregulation in certain forms of psychopathology 8 and maladaptive behaviors. ${ }^{9}$ Furthermore, recent research

\footnotetext{
${ }^{1}$ Department of Psychology, Università Cattolica del Sacro Cuore, Milan, Italy.

${ }^{2}$ Research Unit on Theory of Mind, Department of Psychology, Università Cattolica del Sacro Cuore, Milan, Italy.

${ }^{3}$ Applied Technology for NeuroPsychology Lab, Istituto Auxologico Italiano, Milan, Italy.
} 
suggests the importance of considering person-situation interactionist models that recognize individual differences and emphasize the importance of flexibility in ER. ${ }^{10}$ Through the learning of new and effective ways of regulating their emotions individuals can enhance their emotional lives and protect themselves from the risk of psychopathologies, thus interventions targeting the empowerment of such skill are increasing.

Today new methods and tools to help individuals in managing their affective states are available, including virtual computer-generated settings aimed to manipulate the affective experience ${ }^{11,12}$ and to train specific ER strategies. ${ }^{13-15}$ Among the virtual mediated experiences, videogames are becoming increasingly popular within people's entertainment activities and are attracting the interest of researchers about their opportunities for positive individual functioning. This is consistent with other studies investigating the role of traditional media, which highlighted how different forms of human art and expression have relationships with consumers' social and emotional abilities. ${ }^{16-18}$

Overcoming the typical "good-bad" dichotomy in videogame research, interest is growing toward a deep understanding of the effects of videogames on the development of several abilities, such as cognitive ${ }^{19,20}$ and social ones. ${ }^{21,22}$ To examine the potential positive and negative outcomes of gaming, it is important to go beyond a "cause and effect" approach and to consider a comprehensive gamer-centered approach. Research has recently explored the positive effects of videogames on players' wellbeing, ${ }^{23,24}$ in terms of inducing positive emotions, ${ }^{25}$ improving mood and decreasing stress,${ }^{26}$ contributing to emotional stability, ${ }^{27}$ and promoting engaging, self-actualizing experiences such as psychological flow. ${ }^{28,29}$ Nevertheless, the effects of videogames in terms of ER are gaining attention, but have not yet received systematic in-depth analysis in literature.

Even if videogames may not address the complexity of the human emotional experience, several aspects of videogames are interesting for the promotion of ER. Following the psychological taxonomy of videogames' characteristics proposed by King et al., ${ }^{30}$ it is possible to recognize at least three main opportunities. The first is related to manipulation and control features, including various functions in a videogame that directly relate to the player's sense of mastery and control over the game, such as being able to save progress to correct mistakes, and the ability to simultaneously manage numerous resources. The controlled exposure to negative emotional stimuli may trigger and train reappraisal abilities, which is a key aspect to mature ER. Such property is certainly typical of videogames in which emotional stimuli are repeated and controlled by the player's activity. ${ }^{24,31}$ Furthermore, specific positive effects can emerge also by negative emotions experienced while playing games, such as frustration related to gameplay difficulty or sadness and fear related to tragic or horror contents. ${ }^{32}$ This is related to the interactivity of videogames, which has been labeled as the "art of failure" because it moves the player to constantly manage to overcome obstacles within the game instances. ${ }^{33}$ Thanks to their interactive nature, videogames allow the player to deal continuously and directly with what generates the emotional response and to recover from failure.

The second aspect is related to narrative and identity features that refer to the role of storytelling as a means of immersing the player in the videogame and to the ways the player can take on another identity in the game. Indeed, games can feature a wide range of emotional stimuli most of them integrated within complex and thought-provoking narratives. For example, role-playing games (RPG) allow gamers to select and modify several character features so that playing is associated with the ability to move fluidly among different opportunities for emotional experiences, reappraising them and learning new ways of dealing with negative emotions or disturbing events. ${ }^{24}$ Customization features make possible the creation of a complexity of emotional narratives that are specifically tailored to an individual's own personality and desire ${ }^{34}$ such as, acting as a good or evil person or taking choices different from real life. Furthermore, a player's avatar represents a powerful resource for self-representation and identity play ${ }^{35}$ and the embodied experience of using an avatar can influence the individual's self-perception, ${ }^{36-38}$ the enactment of ER strategies and even the person's behavior. ${ }^{39-41}$ As Gaetan et al. ${ }^{42(\mathrm{p} 344)}$ write, avatars constitute an extraordinary tool for learning and improving ER: "the avatar's evolution in the virtual environment may help mediate adolescents' problematic emotional experiences to give them meaning and enable their appropriation. As such, videogames may act as a medium for projecting and experiencing one's emotional life by staging the emotional self."

The third aspect is related to feedback/reward features that refer to the many ways players are reinforced for skillful play. For example, meta-game rewards, such as points or awards gained upon completion of game milestones, are designed to give players an overall assessment of their mastery over the game itself. According to this approach, games with biofeedback have been recently developed and tested and the main goal was to increase the quality of interaction by modifying the game in real time. This is the case of games such as Nevermind and Deep created to support ER training by encouraging specific patterns of physiological self-regulation. ${ }^{43-45}$

These are some of the aspects specific to videogame technology that could possibly be related to improvements in ER abilities; however, the literature on this topic seems to be still fragmented regarding theoretical approaches, methods, and results. Starting from this background, the present review aims to analyze the state of the art of research on videogames for ER.

This review is restricted to healthy individuals and encompasses all phases of the life span because having clinical disorders related to ER may confound the outcomes that can be different for promotional/preventive interventions compared with treatment ones.

\section{Materials and Methods}

\section{Selection of articles}

Articles were extracted in June 2017 using two academic search engines (PsycINFO \& Scopus) and other articles were suggested by experts in the field. This systematic review was conducted according to PRISMA, a protocol to perform systematic reviews. ${ }^{46}$ Only articles published in peerreviewed journals and conferences, written in English were extracted. Keywords were "emotion regulation" and "videogames." The authors decided to include "positive affect" as a synonym of positive emotion and "management," 
"coping" as synonym of regulation, and to include "mood" as a key word. Also "serious game" word was included, because videogames designed for educational purposes are often labeled in this way in the literature. The search terms were truncated in efforts to include all variations of the word. The following combinatory keyword was used across all selected search engines: emot*\&regulat*\& video game or videogame, emot*\&regulat*\&serious game; emot*\&manag*\&video game or videogame, emot*\&manag*\&serious game; emot*\&cop*\&video game or videogame, emot* \&cop*\&serious game; positive\&affect\&video game or videogame, positive\&affect\&serious game; positive\&emotion\&video game or videogame, positive\&emotion\&serious game; mood\&video game or videogame, mood\&serious game.

In the first round, the titles and abstracts returned $(n=531$, after elimination of 87 duplicates) were screened to exclude: (1) not original research articles (opinions, editorials, and reviews excluded) and (2) studies not focused on ER.

A full-text analysis of the remaining 58 publications was independently performed by three authors (D.V., C.C., S.T.) and each article was assessed based on reliability of its content (background; research method; research results, analysis, and discussions). This step allowed to exclude 35 further publications due to the following reasons: (1) the target was constituted by clinical population (with a specific clinical diagnosis) $(n=23)$; (2) videogames and/or serious games were cited in the text, but not involved in the study $(n=12)$.

Through this screening, twenty-three articles were retained to obtain the final sample. The same coding scheme was used to analyze the retrieved contributions. Table 1 provides a summary of studies' characteristics (year of publication, sample size, age, gender, and outcome) and each contribution was coded according to the following thematic categories: authors and year, research purpose, design, type of game, measures, and findings relevant for ER (Table 2).

Figure 1 contains the PRISMA diagram of the article search, retrieval, and coding process. ${ }^{47}$

\section{Results}

The reviewed studies can be categorized in three main groups, depending on their methodological approaches and on the use of commercial or bespoke games.

\section{Cross-sectional and qualitative studies}

This first group investigated videogaming activity in everyday life and its relationship with a number of constructs, some of them being of interest for ER. In other words, these studies deepened the relationship between ER and commercial videogame play, without experimental settings involving a specific videogame. These studies were mostly cross-sectional research (i.e., employing questionnaires and large samples) and two qualitative studies using focused interviews. For example, Gaetan et al. ${ }^{42}$ conducted a survey to explore videogame playing and its relationships with emotional functioning features, such as ER, emotion intensity, emotion expression, and alexithymia. Authors found that regular gamers regulated their emotions more than irregular gamers did, but also felt emotions more intensely and expressed their emotions less often. Moreover, regular gamers were less prone to be emotionally reactive (alexithymia). Accordingly, another cross-sectional research about videogames and various aspects of psychosocial functioning ${ }^{48}$ found that frequent videogame playing was

Table 1. Studies' Characteristics

\begin{tabular}{|c|c|c|c|}
\hline Characteristic & References & Count & $\%$ \\
\hline Year of publication & & 23 & 100.0 \\
\hline 2007-2010 & $1-7$ & 7 & \\
\hline 2011-2014 & $8-15$ & 8 & 34.8 \\
\hline 2015-June 2017 & $16-23$ & 8 & 34.8 \\
\hline \multicolumn{4}{|l|}{ Sample size } \\
\hline $0-50$ & $13,14,22$ & 3 & \\
\hline $51-100$ & $5,11,12,15,16,17,18$ & 7 & 30.4 \\
\hline $101-200$ & $2,6,7,9,10,19,20,21,23$ & 9 & 39.1 \\
\hline$>201$ & $1,3,4,8$ & 4 & 17.4 \\
\hline \multicolumn{4}{|l|}{ Mean age (years) } \\
\hline$<10$ & 23 & 1 & \\
\hline $10-20$ & $8,16,17,18,19,20,21$ & 7 & 30.4 \\
\hline $20-30$ & $1,2,3,4,5,6,7,9,10,11,12,13,14,15,22$ & 15 & 65.2 \\
\hline \multicolumn{4}{|l|}{ Gender } \\
\hline Both male and female & $1,2,4-13,15-23$ & 21 & 91.3 \\
\hline Male only & 3,14 & 2 & 8.7 \\
\hline \multicolumn{4}{|l|}{ Outcome } \\
\hline Emotion regulation & $3,8,12,14,16,17,18,19$ & 8 & 34.8 \\
\hline Mood repair & $2,6,7,9,10,11,13,15$ & 8 & 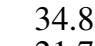 \\
\hline Stress relief & $1,4,5,22,23$ & 5 & 21.7 \\
\hline Anxiety prevention & 20,21 & 2 & 8.7 \\
\hline
\end{tabular}

Reference numbers: 1 , Wood et al. ${ }^{53} ; 2$, Russell Newton ${ }^{56} ; 3$, Wack and Tantleff-Dunn ${ }^{48} ; 4$, Reinecke ${ }^{49} ; 5$, Hussain and Griffiths ${ }^{51} ; 6$, Ferguson and Rueda ${ }^{57} ; 7$, Russoniello et al. ${ }^{26} ; 8$, Seo et al. ${ }^{54} ; 9$, Reinecke et al ${ }^{60}$; 10 , Bowman and Tamburini ${ }^{62}$; 11 , Bowman and Tamburini $^{63}$; 12 , Astor et al. ${ }^{71} ; 13$, Rieger et al ${ }^{58} ; 14$, Lobel et al. ${ }^{55} ; 15$, Rieger et al. ${ }^{59} ; 16$, Cejudo and Latorre ${ }^{67} ; 17$, Rodríguez et al ${ }^{65}$; 18 , Vara et al. ${ }^{66}$; 19 , Gaetan et al. ${ }^{42}$; 20 , Scholten et al. ${ }^{69} ; 21$, Schoneveld et al. ${ }^{70} ; 22$, Kuo et al. ${ }^{52} ; 23$ Hinkley et al. ${ }^{50}$ 


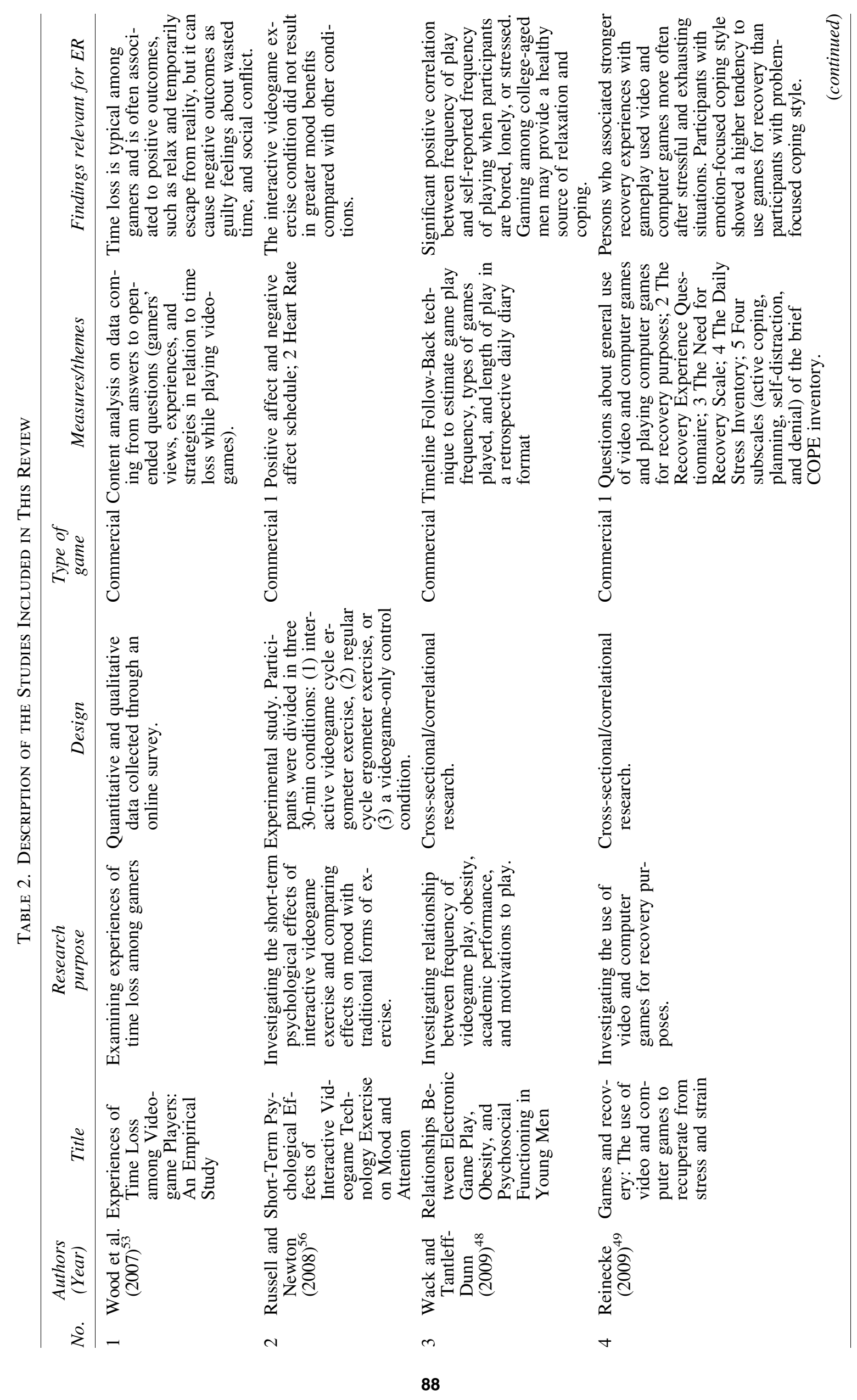




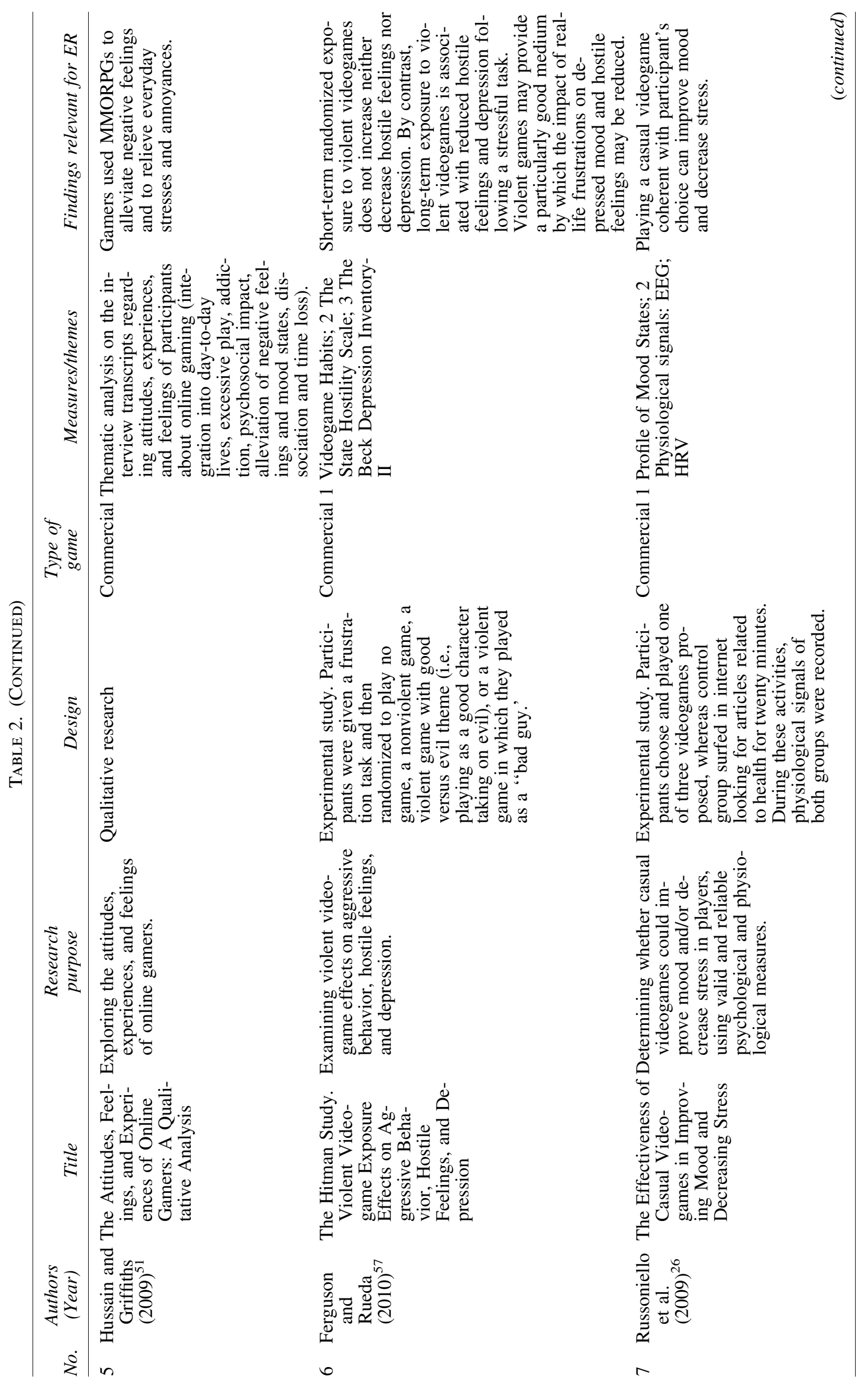




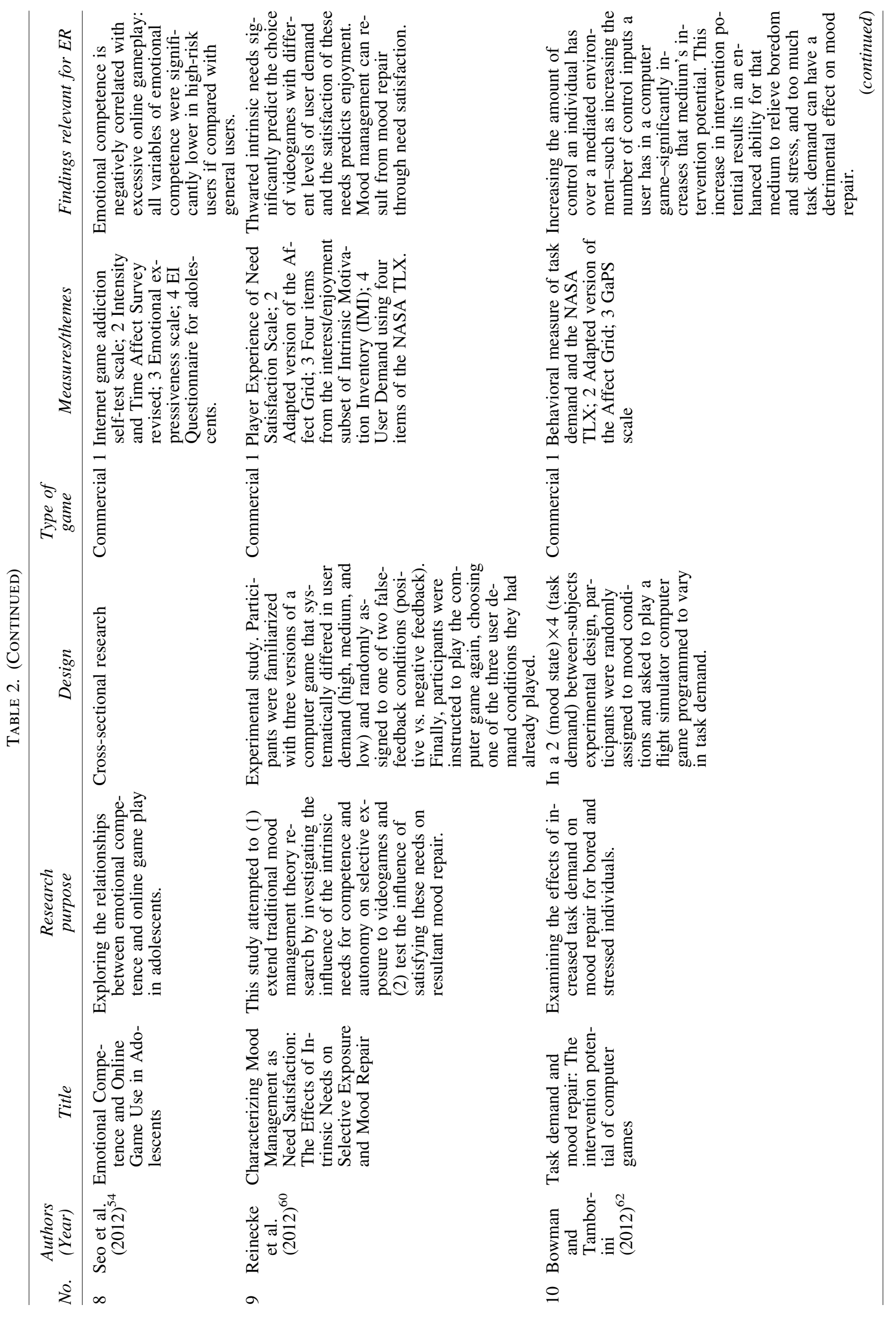




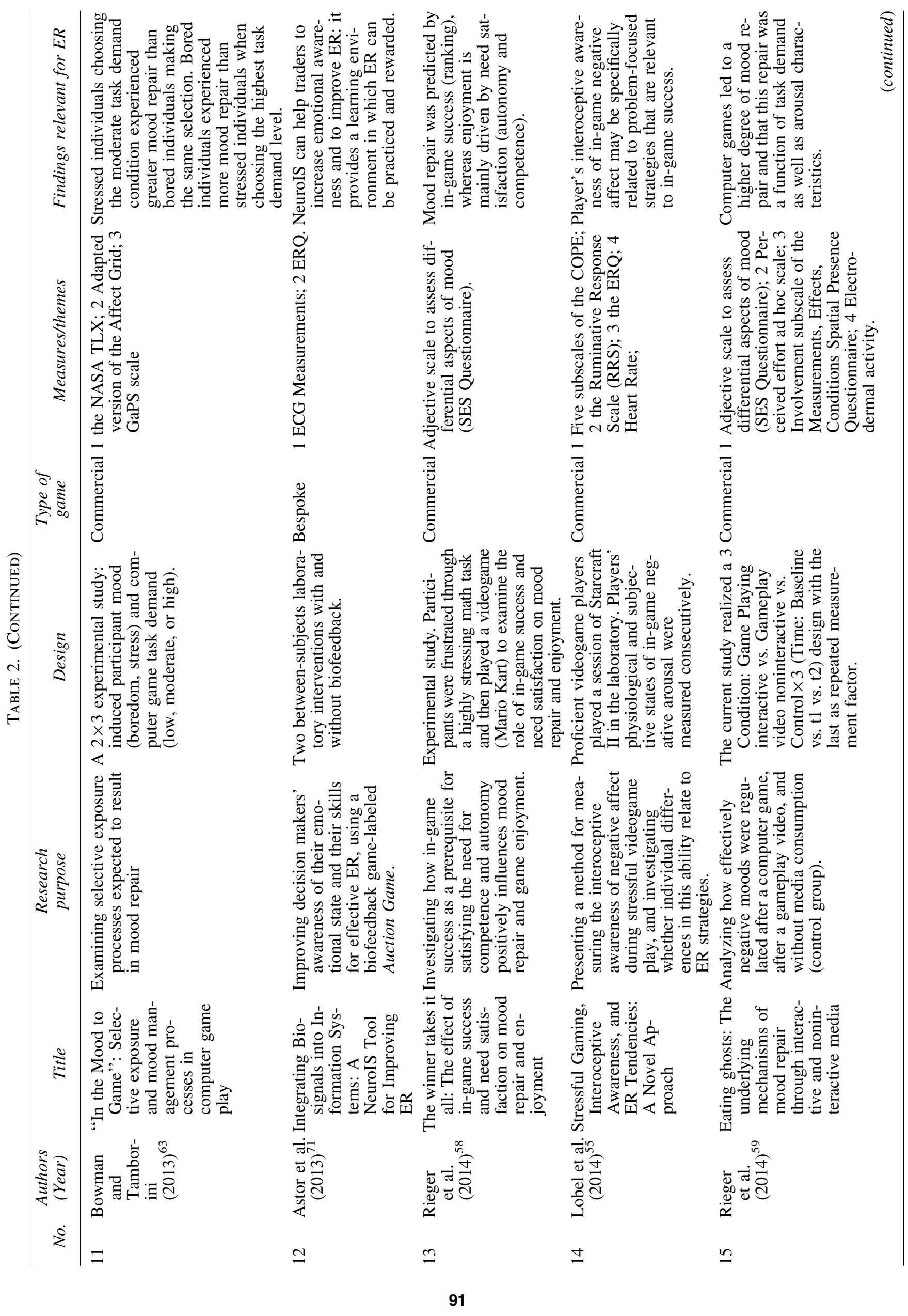




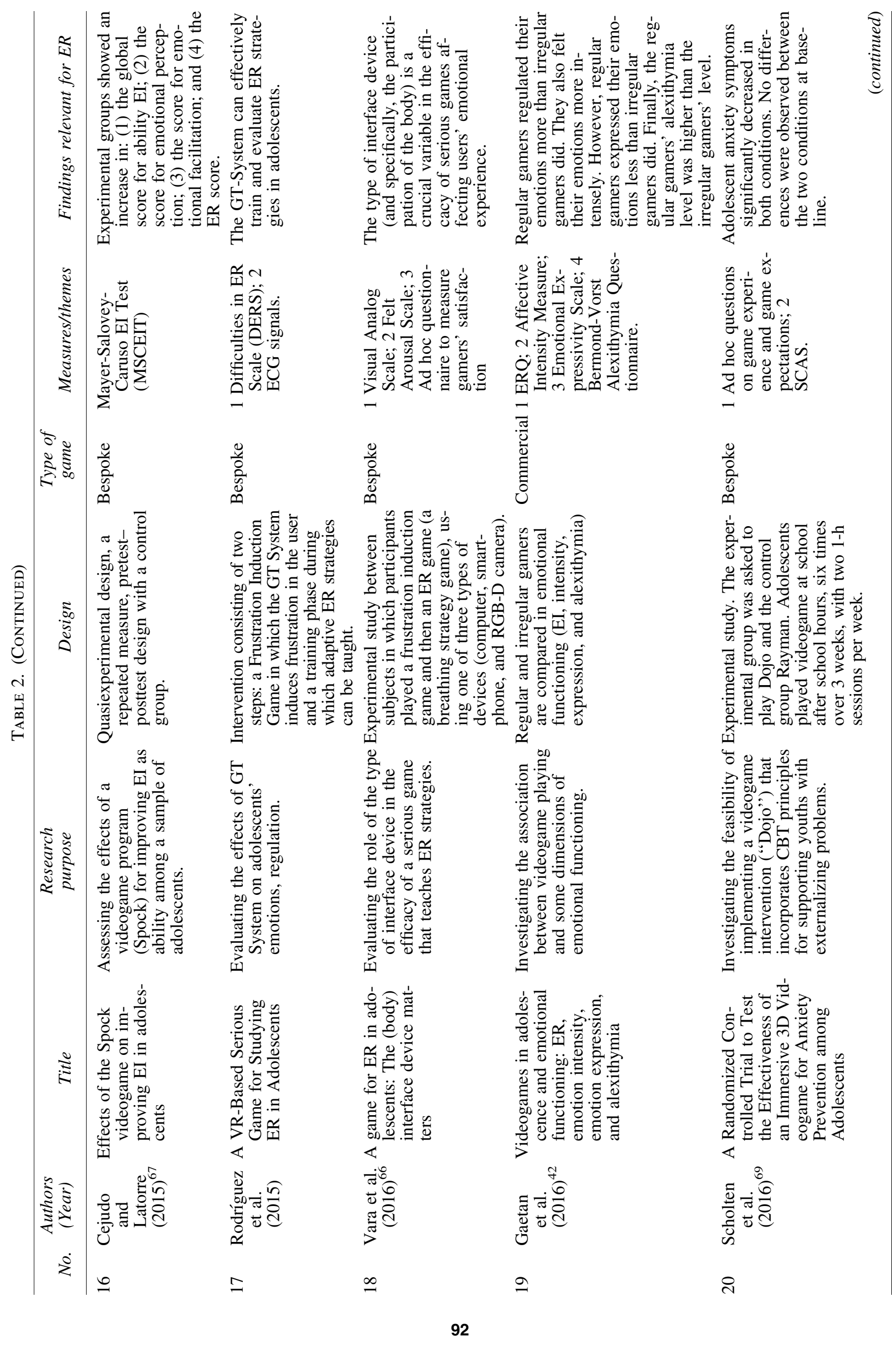




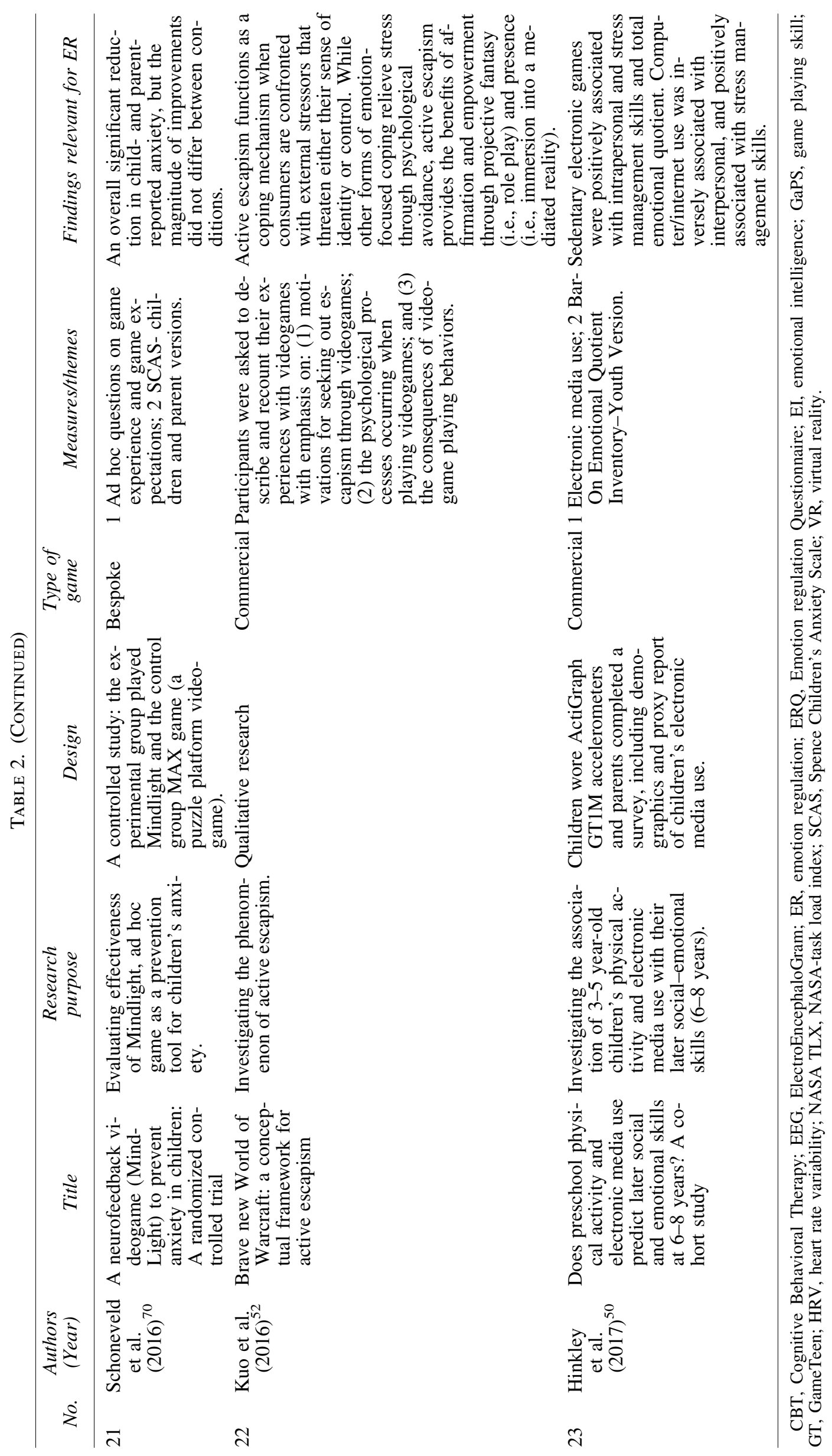




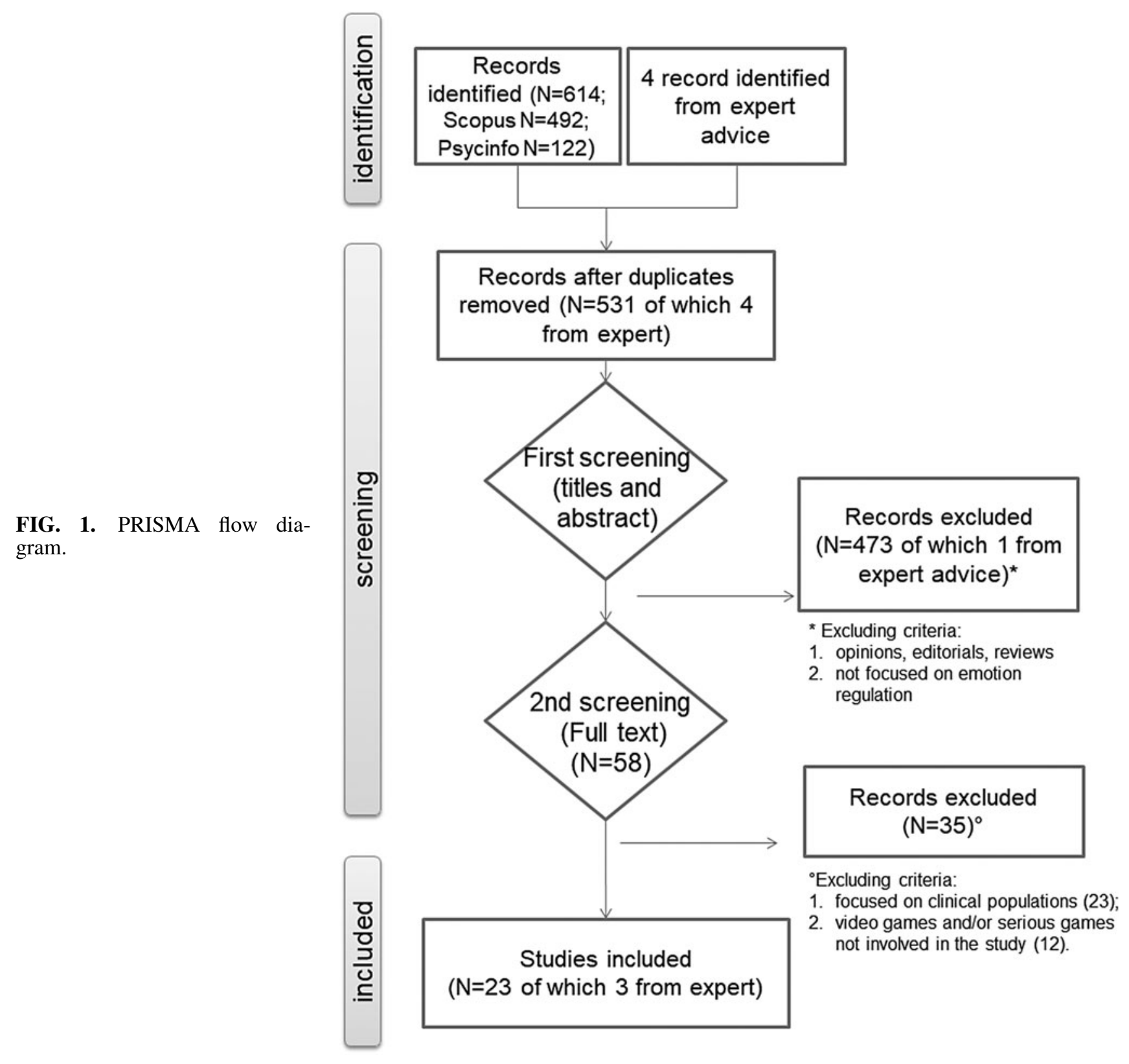

positively related to relaxation and coping. Reinecke ${ }^{49}$ found that videogames are systematically used to recover from stressful situations: specifically, the tendency to do so was stronger in people with emotion focused than problem focused coping style. Hinkley et al. ${ }^{50}$ performed a longitudinal study to investigate the relationship among electronic media use of children (parent reported) and their social-emotional skills: results showed that videogames' play was positively associated with intrapersonal abilities, stress management skills, and global emotional quotient. Moreover, qualitative research on the topic ${ }^{51}$ confirmed that gamers play also to alleviate and manage negative feelings; in terms of "escapism," this could be related to videogames' properties that promote projective fantasy (e.g.: role play) and presence/ immersion (e.g.: feeling inside another reality). ${ }^{52}$ In a different context, Wood et al. ${ }^{53}$ studied the phenomenon of time loss among videogame players. Indeed, the strong engagement in videogaming often leads to not acknowledging time passing. This experience of full absorption in an activity is enhanced by particular structural characteristics of games, such as their complexity and multiplayer interactions, and not with individual characteristics of gamers, such as their gender or age, or frequency of play. From the point of view of ER, this specific feature of videogame playing is related to both positive (e.g., relaxation, escaping from stressful reality) and negative aspects (e.g., guilty feelings about perceived time waste). However, if ER can benefit from regular videogame play, it could be negatively influenced by excessive videogame use: this is what emerged from a cross-sectional research ${ }^{54}$; moreover, sex differences (i.e., females being more able to regulate emotions) disappeared among highrisk gamers. 


\section{Experimental studies on videogame experience for ER}

This group of studies focused on experimental research of different facets of videogame playing experience, providing results interesting for the topic of ER.

The association between in-game experience-measured both with physiological and subjective measures - and realworld regulation strategies have been explored by a recent study by Lobel et al. ${ }^{55}$ Authors observed a positive relation between in-game interoceptive awareness and the selfreported tendency to actively seek a resolution to negative affect. Specifically, the interoceptive awareness of negative affect during stressful game play appeared uniquely related to regulatory strategies that are more problem focused than emotion focused and that are relevant for in-game success. This result opened the doors to the possibility that specific ER skills may be engaged and even trained during stressful videogame play, as in the case of recent biofeedbackintegrated videogames that will be presented within the ER interventions with serious games paragraph.

Other studies investigated how videogames can be used as a resource for mood repair or a well-established function that implies distraction from negative mood and modification of unpleasant arousal states. Russoniello et al. ${ }^{26}$ in a randomized controlled study tested and confirmed the effect of three popular casual videogames on mood and stress. Specifically, authors used different measures to assess change in mood and stress, and electroencephalography and heart rate variability changes corroborated findings on psychological reports. Even if all three games had different yet complimentary mood lifting effects, findings have implications related to the potential development of prescriptive interventions using casual videogames to prevent and treat stressrelated disorders. A study with college students recognized that only the interactivity feature of videogame could offer a potential for interventions aimed to elicit affective benefits as interactive videogames resulted not more effective in repairing mood over other forms of exercise. ${ }^{56}$

Another experiment showed that even violent videogames could offer opportunities for mood repair. Ferguson and Rueda $^{57}$ showed that the use of violent videogames reduces depression and hostile feelings in players. Violent games may provide mechanisms through which players can assert control over a virtual environment both by aggressively demonstrating dominance or acting clear goal-directed behavior. Thus, the author concluded that even violent games may provide, at least for some individuals, a particularly good medium to reduce the impact of real-life frustrations on depressed mood and hostile feelings.

Other recent studies deeply understood the mechanisms and opportunities of videogames for mood repair. Rieger et al. ${ }^{58,59}$ explored in a laboratory setting how in-game success, as a prerequisite for satisfying the need for competence and autonomy, positively influences mood repair and game enjoyment. With the same approach, Reinecke et al. ${ }^{60}$ extended traditional mood management theory research by investigating the influence of the intrinsic needs for competence and autonomy on selective exposure to videogames and tested the influence of satisfying these needs on resultant mood repair. Thwarted intrinsic needs significantly predict the choice of videogames with different levels of user demand and the satisfaction of these needs predicted enjoyment. This result is coherent with recent findings indicating that impedances of player competence satisfaction increase cognitive, affective, and behavioral aspects of aggression. ${ }^{61}$

Bowman and Tamborini extended this line of research with two experimental studies. ${ }^{62,63}$ First, they varied the levels of task demand in a computer game to examine mood repair for bored and stressed individuals. Results confirmed that the increasing of the amount of control an individual has over a mediated environment significantly enhances individual's ability to relieve boredom and stress, but too much task demand is detrimental to mood repair. Second, they investigated the effect of experimentally induced noxious mood states on subsequent selective exposure to computer games known to differ in task demand, as well as the resultant mood repair from these task demand selections. Participants preferred moderate task demand to high and low task demand, and this preference was stronger for stressed participants.

\section{ER intervention with serious games}

Finally, the last group of studies included interventions specifically focused on the use of videogames to improve affect regulation abilities. All these studies involved the use of videogames created to achieve specific objectives; these bespoke games include serious games or games with specific educational purposes, and games specifically developed to perform experimental studies.

One research group developed and tested a number of videogames specifically devoted to the empowerment of ER abilities in adolescents. This was done because deficits in ER during adolescence may evolve into mental health problems later in life. A virtual multiplatform system (GameTeen) based on Ecological Momentary Assessment, ${ }^{64}$ which allows the therapist to monitor the adolescent's emotional status day by day, was implemented and tested. The system contains mood-induction arcade games (joy and frustration) and mini games to train and evaluate ER strategies (for example, a moving feather that helped the player to control his own breath to promote relaxation). In the end, GameTeen was evaluated as pleasant, usable, and appeared more effective to teach ER than control, paper-based counterparts. In the context of subsequent evaluations of the same system ${ }^{65}$ both psychophysiological variables (heart rate) and questionnaire data demonstrated that the game could induce frustration, and then promote effective regulation of the previously induced negative emotion. Another study ${ }^{66}$ demonstrated that the use of specific interface devices significantly impacted the effectiveness of the GameTeen tool (i.e., personal computer and tablet were more effective than an RGB-D camera). Similar results were obtained by Cejudo and Latorre ${ }^{67}$ while evaluating the effectiveness of the videogame Spock in promoting emotional intelligence, namely a complex construct involving the abilities of perceiving, recognizing, generating, and regulating emotions so to sustain wellbeing and intellectual growth. ${ }^{68}$

Scholten et al. ${ }^{69}$ tested Dojo, a biofeedback videogame created for anxiety prevention: in this case, anxiety symptoms equally decreased both in the experimental and the control group (playing the commercial videogame Rayman 2: The Great Escape, Ubisoft, 1999). Latent growth curve models revealed a steeper decrease of personalized anxiety 
symptoms (not of total anxiety symptoms) in the Dojo condition compared with the control condition. Another randomized controlled trial focused on anxiety prevention among childre ${ }^{70}$ involving Mindlight, a neurofeedback videogame for anxiety prevention, or Max and the Magic Marker (Press Play, The Games Company, 2010), a commercial videogame as control. Results revealed reduction in children and parents' reported anxiety, but the magnitude of effects did not differ between conditions.

In a different context, Astor et al. ${ }^{71}$ hypothesized that some videogame playing aspects could be useful to promote emotion management in traders and investors, who may be often negatively influenced by emotions when engaged in financial decisions. Authors designed and implemented a serious game-based NeuroIS tool that continuously displayed the player's individual emotional state, through biofeedback, and adapted the gaming environment to the players' physiological responses. Positive results from two laboratory studies showed that biofeedback technology could be used effectively in the design of finance-centered information systems.

\section{Discussion}

The present review highlighted a high variety of studies investigating the relationship among videogame playing and ER abilities. A group of studies employed cross-sectional and qualitative research finding consistent results: videogame playing may enhance emotional intelligence and the mastering of ER strategies. ${ }^{42,48,54}$ Nevertheless, excessive videogame playing may negatively influence such competences. ${ }^{54}$ This is in line with other studies ${ }^{72}$ showing a curvilinear relationship between videogame playing and mental health outcomes, with "moderate" gamers showing better mental health and psychosocial functioning.

Thanks to the regular game activity, gamers may cope with emotional stimuli that they cannot normally experience in real life. Thus gamers can learn adaptive ER strategies. Additionally, the identification with a character constitutes a critical affordance to enhance emotional skills. ${ }^{42}$

Exploring gaming motivations, several studies agree on the fact that people usually play videogames to recover from stress and negative emotions. ${ }^{49,51,52}$ This can be regarded as the simplest way videogames can interact with players' emotions and mood, and it is the specific phenomenon investigated by mood repair. ${ }^{58,59}$ According to the mood management perspective, ${ }^{73}$ people select entertainment for pleasure-seeking (or stress avoidance) purposes. Moreover, the selection of meaningful entertainment experiences is associated with appreciation; thus, games also provide players with experiences that are not only enjoyable but also potentially deeply enriching. ${ }^{74}$

Experimental studies extended the traditional mood management theory research by discovering that not only the selection of videogames coherent with user needs is important but that also the satisfaction of the intrinsic needs for competence and autonomy influences enjoyment and mood repair. ${ }^{60,62,63}$ Furthermore, experimental studies showed that violent games may provide a mechanism through which players can assert control over a virtual environment thus demonstrating dominance and clear goal-directed behavior and reducing negative feelings. ${ }^{57}$
As narrative, interactive, and symbolic media, videogames could represent an affordance for the design of digital interfaces explicitly devoted to the improvement of ER. For this reason, serious games have been developed and tested exactly with this aim and they generally give encouraging results. For example, games designed to improve ER abilities such as GameTeen and Spock succeeded in their aim $^{65-67}$; similarly, a game to improve emotion management in adult financial experts obtained positive results. ${ }^{71}$ However, when serious games designed and developed as ER tools are rigorously evaluated in randomized controlled trials they obtain only partial results. ${ }^{69,70}$ Although encouraging, ER intervention with serious games are characterized by the use of different games, samples, and ER-related techniques. They typically compare the game with no treatment at all, or they compare different devices. Consequently, more effort is required to define specific guidelines for this field of intervention.

This review shows that the use of commercial and bespoke videogames offers different potentialities for ER. Specifically, the continual, passionate yet not-excessive experience with personally relevant videogames represents a concrete resource to experiment with rich emotional states and exercise coping abilities within secure, simulated contexts. This highlights that commercial videogames offer several advantages in terms of interaction, control, and narrative features; however, further research is needed to understand what factors may impact on the ER enhancement process. For example, videogame opportunities are certainly differently distributed and incarnated by different videogame genres (e.g.: RPGs and Adventures are typically more based on narrative than other genres); moreover, videogame players' expectations may influence the videogame experience (for example passionate gamers could expect more emotional intensity or be more positive when dealing with well-known games they already love and respect than with educational products). On the other side, controlled trials with bespoke games (serious games or games developed for experimental purposes) showed that their actual capacity to empower ER is controversial. Indeed, these games were often limited-time experiences and not able to include all the typical complex properties (e.g., avatar identification; sophisticated immersion into narrative) of commercial games.

The principal limitation of the present review is that is represented by its own inclusion/exclusion criteria. To focus on the specific phenomenon of ER as a healthy life skill, the researchers decided to exclude studies involving people suffering from physical or psychological disorders, which could have possibly influenced the results. A recent longitudinal study aimed to understand the effects of videogames on psychosocial development ${ }^{75}$ found that gaming frequency in healthy children was associated with an increase in internalizing problems, such as anxiety and depressive symptoms. This result calls for the need to adopt a bidirectional analysis' perspective between pre-existing psychosocial and clinical characteristics of gamers and positive or negative outcomes. Thus, future reviews may focus on clinical populations, especially involving those disorders related to emotion expression and regulation (e.g., anxiety disorders, autism, etc.), to see whether videogames improving ER may be more or less effective when integrated with psychotherapy. 


\section{Conclusion}

What emerges from this review is that videogames should not be considered only as entertaining, interactive devices; rather, they are fully suitable to be recognized in their complexity and richness as communicational and narrative media with legitimate application in understanding and influencing ER.

The information reported by the present review of experimental studies constitutes an initial guideline both for designers and professionals. On the one hand, this review gives suggestions for the design of future serious games for emotional/social abilities: bespoke game designers should take advantage of complex fictional properties of videogames. On the other hand, psychological interventions for game-based ER empowerment could consider the possibility of building intervention protocols around commercial games selected on the basis of specific properties. Thus, the challenge for educational and psychological intervention is to exploit the emotional and affective affordances of videogames, so to make use of the rich properties of these simulated experiences, as previously done with other traditional media. ${ }^{76,77}$

\section{Authors Disclosure Statement}

No competing financial interests exist.

\section{References}

1. Thompson RA. Emotion regulation: A theme in search of definition. Monogr Soc Res Child Dev 1994; 59:25.

2. Gross JJ. The emerging field of emotion regulation: An integrative review. Rev Gen Psychol 1998; 2:271-299.

3. Gross JJ, Thompson RA. Emotion Regulation: Conceptual and Empirical Foundations. In: Gross JJ, ed. Handbook of Emotion Regulation. New York: Guilford Press; 2007:3-24.

4. Koole SL. The psychology of emotion regulation: An integrative review. Cogn Emotion 2009; 23:4-41.

5. Gross JJ, Muñoz RF. Emotion regulation and mental health. Clin Psychol Sci Pract 1995; 2:151-164.

6. Balzarotti S, Biassoni F, Villani D, et al. Individual differences in cognitive emotion regulation: implications for subjective and psychological well-being. J Happiness Stud 2016; 17:125-143.

7. Murray SL. Regulating the risks of closeness a relationshipspecific sense of felt security. Curr Dir Psychol Sci 2005; 14:74-78.

8. Aldao A, Nolen-Hoeksema S, Schweizer S. Emotionregulation strategies across psychopathology: A metaanalytic review. Clin Psychol Rev 2010; 30:217-237.

9. Harrison A, Sullivan S, Tchanturia K, Treasure J. Emotional functioning in eating disorders: Attentional bias, emotion recognition and emotion regulation. Psychol Med 2010; 40:1887-1897.

10. Bonanno GA, Burton CL. Regulatory flexibility. Perspect Psychol Sci 2013; 8:591-612.

11. Riva G, Mantovani F, Capideville CS, et al. Affective interactions using virtual reality: The link between presence and emotions. Cyberpsychol Behav 2007; 10:45-56.

12. Villani D, Riva G. Does interactive media enhance the management of stress? Suggestions from a controlled study. Cyberpsychol Behav Soc Network 2012; 15:24-30.

13. Carissoli C, Villani D, Riva G. Does a meditation protocol supported by a mobile application help people reduce stress? Suggestions from a controlled pragmatic trial. Cyberpsychol Behav Soc Network 2015; 18:46-53.

14. Gaggioli A, Pallavicini F, Morganti L, et al. Experiential virtual scenarios with real-time monitoring (interreality) for the management of psychological stress: A block randomized controlled trial. J Med Internet Res 2014; 16:e167.

15. Tunney C, Cooney P, Coyle D, O'Reilly G. Comparing young people's experience of technology-delivered v. faceto-face mindfulness and relaxation: Two-armed qualitative focus group study. Br J Psychiatry 2016. DOI:10.1192/ bjp.bp.115.172783.

16. Thoma MV, Scholz U, Ehlert U, Nater UM. Listening to music and physiological and psychological functioning: The mediating role of emotion regulation and stress reactivity. Psychol Health 2012; 27:227-241.

17. Bartsch A, Vorderer P, Mangold R, Viehoff R. Appraisal of emotions in media use: Toward a process model of metaemotion and emotion regulation. Media Psychol 2008; 11 : 7-27.

18. Cheetham M, Hänggi J, Jancke L. Identifying with fictive characters: Structural brain correlates of the personality trait "fantasy." Soc Cogn Affect Neurosci 2014; 9:1836-1844.

19. Green CS, Bavelier D. Effect of action video games on the spatial distribution of visuospatial attention. J Exp Psychol Hum Percept Perform 2006; 32:1465-1478.

20. Adachi PJC, Willoughby T. More than just fun and games: The longitudinal relationships between strategic video games, self-reported problem solving skills, and academic grades. J Youth Adolescence 2013; 42:1041-1052.

21. Greitemeyer T, Osswald S, Brauer M. Playing prosocial video games increases empathy and decreases schadenfreude. Emotion 2010; 10:796-802.

22. Gentile DA, Anderson CA, Yukawa S, et al. The effects of prosocial video games on prosocial behaviors: International evidence from correlational, longitudinal, and experimental studies. Pers Soc Psychol Bull 2009; 35:752-763.

23. Jones CM, Scholes L, Johnson D, et al. Gaming well: Links between videogames and flourishing mental health. Front Psychol 2014; 5:260.

24. Granic I, Lobel A, Engels RCME. The benefits of playing video games. Am Psychol 2014; 69:66-78.

25. Osmanovic S, Pecchioni L. Beyond entertainment: Motivations and outcomes of video game playing by older adults and their younger family members. Games Cult 2015; 11:1-20.

26. Russoniello CV, O'Brien K, Parks JM. The effectiveness of casual video games in improving mood and decreasing stress. J Cyber Ther Rehabil 2009; 2:53-66.

27. Przybylski AK, Weinstein N, Murayama K, et al. The ideal self at play: The appeal of video games that let you be all you can be. Psychol Sci 2012; 23:69-76.

28. Neri de Souza F. Science education with and through ICT: Curriculum design and questioning to promote active learning. In: Fonseca D, Redondo E, eds. Handbook of Research on Applied E-Learning in Engineering and Architecture Education. Hershey: IGI Global; 2016; 1:133-158.

29. Cowley B, Charles D, Black M, Hickey R. Toward an understanding of flow in video games. Comput Entertain 2008; 6:20:1-20:27.

30. King D, Delfabbro P, Griffiths M. Video game structural characteristics: A new psychological taxonomy. Int J Mental Health Addict 2010; 8:90-106.

31. Gee JP. What video games have to teach us about learning and literacy. Comput Entertainment 2003; 1:20. 
32. Triberti S. This drives me nuts! In: Villani D, Cipresso P, Gaggioli A, Riva G, eds. Integrating Technology in Positive Psychology Practice. Hershey: IGI Global; 2016: 266-289.

33. Juul J. The Art of Failure. An Essay on the Pain of Playing Videogames. Cambridge: MIT Press; 2013.

34. Triberti S, Villani D, Riva G. Moral positioning in video games and its relation with dispositional traits: The emergence of a social dimension. Comput Human Behav 2015; 50:1-8.

35. Villani D, Gatti E, Confalonieri E, Riva G. Am I my avatar? A tool to investigate virtual body image representation in adolescence. Cyberpsychol Behav Soc Network 2012; 15:435-440.

36. Villani D, Gatti E, Triberti S, et al. Exploration of virtual body-representation in adolescence: The role of age and sex in avatar customization. SpringerPlus 2016; 5:740.

37. Mancini T, Sibilla F. Offline personality and avatar customisation. Discrepancy profiles and avatar identification in a sample of MMORPG players. Comput Human Behav 2017; 69:275-283.

38. Triberti S, Durosini I, Aschieri F, et al. Changing avatars, changing selves? The influence of social and contextual expectations on digital rendition of identity. Cyberpsychol Behav Soc Network 2017; 20:501-507.

39. Fox J, Bailenson JN, Tricase L. The embodiment of sexualized virtual selves: The proteus effect and experiences of self-objectification via avatars. Comput Hum Behav 2013; 29:930-938.

40. Wrzesien M, Rodríguez A, Rey B, et al. How the physical similarity of avatars can influence the learning of emotion regulation strategies in teenagers. Comput Hum Behav 2015; 43:101-111.

41. Schek EJ, Mantovani F, Realdon O, et al. Positive technologies for promoting emotion regulation abilities in adolescents. eHealth 2017; 181:169-174.

42. Gaetan S, Bréjard V, Bonnet A. Video games in adolescence and emotional functioning: Emotion regulation, emotion intensity, emotion expression, and alexithymia. Comput Hum Behav 2016; 61:344-349.

43. Hilborn O, Cederholm H, Eriksson J, Lindley C. A biofeedback game for training arousal regulation during a stressful task: The space investor. In: Lecture Notes in Computer Science (Including Subseries Lecture Notes in Artificial Intelligence and Lecture Notes in Bioinformatics). Berlin, Heidelberg: Springer; 2013:403-410.

44. Lobel A, Gotsis M, Reynolds E, et al. Designing and Utilizing Biofeedback Games for Emotion Regulation. Proceedings of the 2016 CHI Conference Extended Abstracts on Human Factors in Computing Systems-CHI EA'16. 2016:1945-1951.

45. van Rooij M, Lobel A, Harris O, et al. DEEP: A biofeedback virtual reality game for children at-risk for anxiety. Proceedings of the $2016 \mathrm{CHI}$ Conference Extended Abstracts on Human Factors in Computing Systems 2016: 1989-1997.

46. Liberati A, Altman DG, Tetzlaff J, et al. Annals of internal medicine academia and clinic the PRISMA statement for reporting systematic reviews and meta-analyses of studies that evaluate health care interventions. Ann Intern Med 2009; 151:W65-W94.

47. Moher D, Liberati A, Tetzlaff J, et al. Preferred reporting items for systematic reviews and meta-analysis: The PRISMA statement. Ann Intern Med 2009; 151:264-269.
48. Wack E, Tantleff-Dunn S. Relationships between electronic game play, obesity, and psychosocial functioning in young men. Cyberpsychol Behav Soc Network 2009; 12:241.

49. Reinecke L. Games and recovery: The use of video and computer games to recuperate from stress and strain. J Media Psychol 2009; 21:126-142.

50. Hinkley T, Timperio A, Salmon J, Hesketh K. Does preschool physical activity and electronic media use predict later social and emotional skills at 6 to 8 years? A cohort study. J Phys Activity Health 2017; 14:308-316.

51. Hussain Z, Griffiths MD. The attitudes, feelings, and experiences of online games: A qualitative analysis. CyberPsychol Behav 2009; 12:747-753.

52. Kuo A, Lutz RJ, Hiler JL. Brave new World of Warcraft: A conceptual framework for active escapism. J Consum Mark 2016;33:498-506.

53. Wood RTA, Griffiths MD, Parke A. Experiences of time loss among videogame players: An empirical study. Cyberpsychol Behav 2007; 10:38-44.

54. Seo M, Kang HS, Chae S-M. Emotional competence and online game use in adolescents. Comput Inform Nurs 2012; 30:640-6-8.

55. Lobel A, Granic I, Engels RCME. Stressful gaming, interoceptive awareness, and emotion regulation tendencies: A novel approach. CyberPsychol Behav Soc Network 2014; 17:222-227.

56. Russell WD, Newton M. Short-term psychological effects of interactive video game technology exercise on mood and attention. Educ Technol Soc 2008; 11:294-308.

57. Ferguson CJ, Rueda SM. The Hitman study: Violent video game exposure effects on aggressive behavior, hostile feelings, and depression. Eur Psychol 2010; 15:99-108.

58. Rieger D, Wulf T, Kneer J, et al. The winner takes it all: The effect of in-game success and need satisfaction on mood repair and enjoyment. Comput Hum Behav 2014; 39:281-286.

59. Rieger D, Frischlich L, Wulf T, et al. Eating ghosts: The underlying mechanisms of mood repair via interactive and noninteractive media. Psychol Popular Media Cult 2015; 4 : $138-154$.

60. Reinecke L, Tamborini R, Grizzard M, et al. Characterizing mood management as need satisfaction: The effects of intrinsic needs on selective exposure and mood repair. J Commun 2012; 62:437-453.

61. Przybylski AK, Deci EL, Rigby CS, Ryan RM. Competenceimpeding electronic games and players' aggressive feelings, thoughts, and behaviors. J Pers Soc Psychol 2014; 106:441457.

62. Bowman ND, Tamborini R. Task demand and mood repair: The intervention potential of computer games. New Media Soc 2012; 14:1339-1357.

63. Bowman ND, Tamborini R. "In the Mood to Game": Selective exposure and mood management processes in computer game play. New Media Soc 2013; 17:375-398.

64. Alcañiz M, Rodríguez A, Rey B, Parra E. Using serious games to train adaptive emotional regulation strategies. In: Lecture Notes in Computer Science (Including Subseries Lecture Notes in Artificial Intelligence and Lecture Notes in Bioinformatics). Berlin, Heidelberg: Springer; 2014:541-549.

65. Rodriguez A, Rey B, Vara MD, et al. A VR-based serious game for studying emotional regulation in adolescents. IEEE Comput Graph Appl 2015; 35:65-73.

66. Vara MD, Baños RM, Rasal P, et al. A game for emotional regulation in adolescents: The (body) interface device matters. Comput Hum Behav 2016; 57:267-273. 
67. Cejudo J, Latorre S. Effects of the Spock videogame on improving emotional intelligence in adolescents. Electron $\mathrm{J}$ Res Educ Psychol 2015; 13:319-342.

68. Mayer J, Salovey P. What is emotional intelligence. In: Harper C, ed. Emotional Development and Emotional Intelligence: Eduational Implications. New York: Basic Books; 1997:10-11.

69. Scholten H, Malmberg M, Lobel A, et al. A randomized controlled trial to test the effectiveness of an immersive 3D video game for anxiety prevention among adolescents. PLoS One 2016; 11:e0147763.

70. Schoneveld EA, Malmberg M, Lichtwarck-Aschoff A, et al. A neurofeedback video game (MindLight) to prevent anxiety in children: A randomized controlled trial. Comput Hum Behav 2016; 63:321-333.

71. Astor PJ, Adam MTP, Jerčić P, et al. Integrating biosignals into information systems: A neurois tool for improving emotion regulation. J Manage Inf Syst 2013; 30: 247-278.

72. Allahverdipour H, Bazargan M, Farhadinasab A, Moeini B. Correlates of video games playing among adolescents in an Islamic country. BMC Public Health 2010; 10:1-7.

73. Zillmann D. Mood management in the context of selective exposure theory. Ann Int Commun 2000; 23:103-123.
74. Oliver MB, Bowman ND, Woolley JK, et al. Video games as meaningful entertainment experiences. Psychol Popular Media Cult 2015; 5:1-16.

75. Lobel A, Engels RCME, Stone LL, et al. Video gaming and children's psychosocial wellbeing: A longitudinal study. J Youth Adolesc 2017; 46:884-897.

76. Shimamura AP, Marian DE, Haskins AL. Neural correlates of emotional regulation while viewing films. Brain Imaging Behav 2013; 7:77-84.

77. Suckfüll M. Emotion regulation by switching between modes of reception. In: Shimamura AP, ed. Psychocinematics: Exploring Cognition at the Movies. Oxford: Oxford University Press; 2013:314-336.

Address correspondence to: Daniela Villani, PhD

Department of Psychology Università Cattolica del Sacro Cuore Largo Gemelli Milan 1-20100 Italy

E-mail: daniela.villani@unicatt.it 